\title{
Erratum to: Hypothetical superparamagnetic magnetometer in a pigeon's upper beak probably does not work
}

\author{
Petr Jandačka ${ }^{1,2, a}$, Petr Alexa ${ }^{2,3}$, Jaromír Pištora ${ }^{1}$, and Jana Trojková ${ }^{2}$ \\ 1 Nanotechnology Centre and IT4Innovations Centre VŠB-Technical University of Ostrava, 17. listopadu 15/2172, 70833, Os- \\ trava, Czech Republic \\ 2 Institute of Physics, VŠB-Technical University of Ostrava, 17. listopadu 15/2172, 70833, Ostrava, Czech Republic \\ 3 Institute of Clean Technologies, VŠB-Technical University of Ostrava, 17. listopadu 15/2172, 70833, Ostrava, Czech Republic
}

Original article: Eur. Phys. J. E (2013) 36: 40, DOI: 10.1140/epje/i2013-13040-1

Received 28 June 2013

Published online: 30 August 2013 - (c) EDP Sciences / Società Italiana di Fisica / Springer-Verlag 2013

Equation (5) (derived from the correct eq. (4)) and the related fig. 5 are not accurate, since we approximated the susceptibility $\chi_{\mathrm{sh}} \approx \chi$ and thus neglected the influence of the cluster demagnetization factor $N=1 / 3$ on $\chi_{\mathrm{sh}}$ (this approximation is valid only for $a_{\mathrm{v}}<3 \mathrm{~nm}$ ). The corrected eq. (5) now reads

$$
\Delta t=\frac{36 \eta \mu_{0}}{5 B^{2}}\left(N+\frac{18 k_{B} T}{\mu_{0} P M_{\mathrm{sat}}^{2} \pi a_{\mathrm{v}}^{3}}\right)^{2} \frac{D_{1}^{5}-D_{2}^{5}}{d^{5}} .
$$

The change in the cluster mobility calculated from the corrected eq. (5) can be seen in fig. 5, right panel.
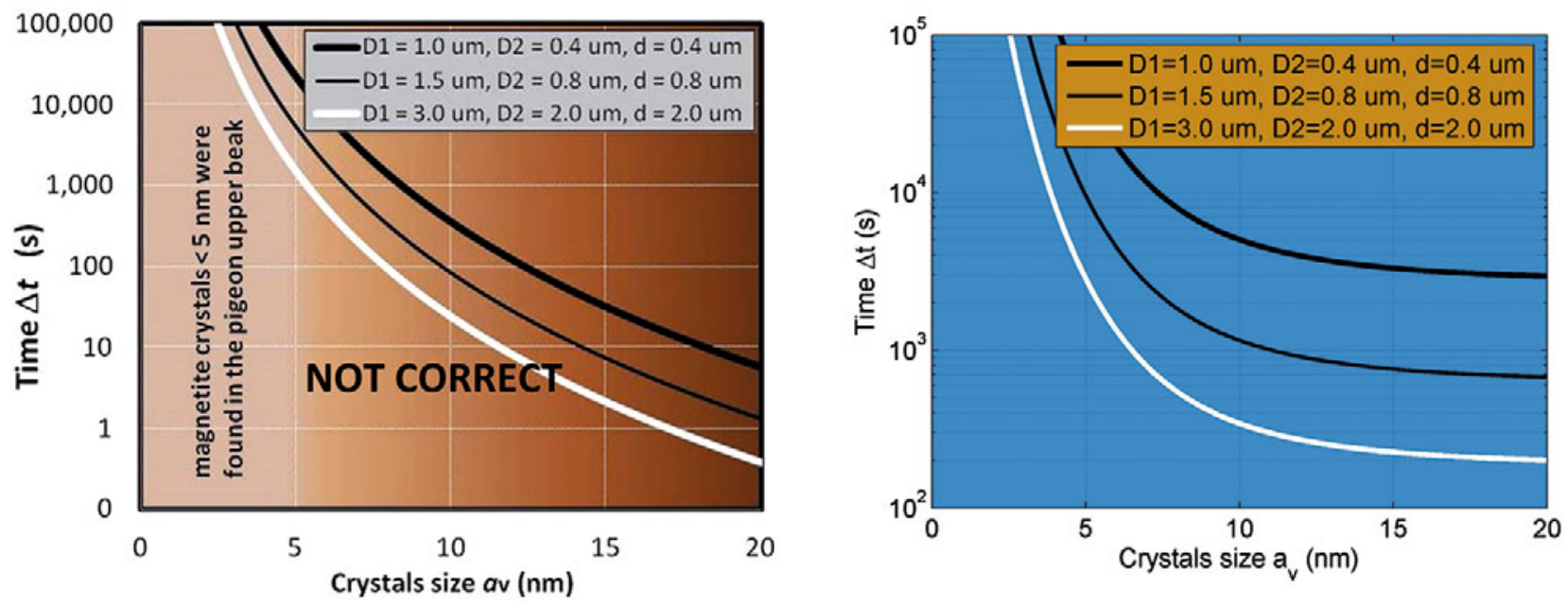

Fig. 5. Mobility of the superparamagnetic magnetite clusters in the cell plasma. $D_{1}$ is the initial distance between two clusters, $D_{2}$ is the final distance between these clusters and $d$ is the size of the clusters. Original uncorrected (left) and corrected (right).

${ }^{a}$ e-mail: petr.jandacka@vsb.cz 
If we assume that the minimum intercluster distance $D_{2}=d$, we can calculate the clusters displacement ratio $D_{1} / D_{2}$ as a function of particle size with average volume $a_{\mathrm{v}}$ for different durations of mutual motion $\Delta t$ from

$$
\frac{D_{1}}{D_{2}}=\left[\frac{5 B^{2} \Delta t}{36 \eta \mu_{0}\left\{N+18 k_{B} T /\left(\mu_{0} P M_{\mathrm{sat}}^{2} \pi a_{\mathrm{v}}^{3}\right)\right\}^{2}}+1\right]^{1 / 5} .
$$

$D_{1} / D_{2}$ as a function of $a_{\mathrm{v}}$ for $\Delta t=10 \mathrm{~s}, 100 \mathrm{~s}$ and $1000 \mathrm{~s}$ is displayed in fig. 6.

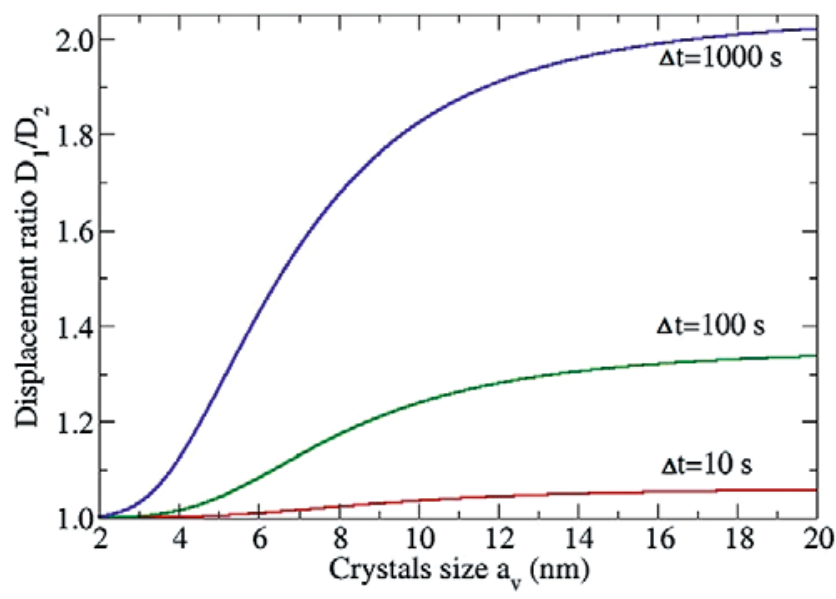

Fig. 6. Displacement ratio $D_{1} / D_{2}$ as a function of $a_{\mathrm{v}}$ for different values of $\Delta t$.

\section{Conclusion}

The superparamagnetic magnetite clusters composed of 15-20 nm crystals could be responsible for the map magnetoreception, but their mobility is slower than originally presented. The conclusions of the original paper remain generally unchanged. 